\title{
Stress associated with onset of recurrent aphthous stomatitis
}

\author{
Abstracted from \\ Huling LB, Baccaglini L, Choquette L, Feinn RS, Lalla RV. \\ Effect of stressful life events on the onset and duration of recurrent aphthous stomatitis. \\ J Oral Pathol Med 2012; 41: 149-152. doi: 10.1111/j.1600-0714.2011.01102.x. Epub 2011 Nov 12. PubMed PMID: 22077475 \\ Address for correspondence: Dr R Lalla, School of Dental Medicine, \\ University of Connecticut Health Center, Farmington, CT, USA. E-mail: lalla@uchc.edu
}

\section{Question: Does stress have an impact on the onset and duration of recurrent aphthous ulceration?}

Design Randomised controlled trial (RCT).

Intervention One hundred and sixty patients participating in a RCT in which they were randomised to multivitamins $(n=81)$ or placebo $(n=79)$ completed ulcer diaries and a weekly telephone survey by calling an automated interactive voice response (IVR) system. Stressful events were quantified using the validated Recent Life Changes Questionnaire (RLCQ) and were classified as mental or physical stressors.

Outcome measure Onset and duration of RAS episodes

Results Stressful life events were significantly associated with the onset of RAS episodes $(P<0.001)$, but not with the duration of the RAS episodes. Experiencing a stressful life event increased the odds of an RAS episode by almost three times $(\mathrm{OR}=2.72 ; 95 \% \mathrm{Cl}=2.04$ 3.62). When controlled for each other, mental stressors had a larger effect $(\mathrm{OR}=3.46,95 \% \mathrm{Cl}=2.54-4.72)$ than physical stressors $(\mathrm{OR}=$ $1.44 ; 95 \% \mathrm{Cl}=1.04-1.99)$ on the occurrence of RAS episodes. RAS episodes did not occur more frequently or last longer with increasing stress severity.

Conclusions In patients with a history of RAS, stressful events may mediate changes involved in the initiation of new RAS episodes. Mental stressors are more strongly associated with RAS episodes than physical stressors.

\section{Commentary}

The study used data from 160 subjects with a history of recurrent aphthous stomatitis (RAS) who participated in a randomised clinical trial evaluating the effect of multivitamin supplementation on the incidence and duration of RAS over a year period. ${ }^{1}$

The subjects in the trial had to report and to describe any stressful event that had been experienced during the week prior to the RAS.

The questionnaire used was a validated tool to quantify stressful events: Recent Life Changes Questions questionnaire (RLCQ). The stressful events were classified as mental or physical stressors.
The questionnaire classifies different life events and assigns them a numerical value.

From 160 subjects, the study collected information reporting 784 stressful events and 600 RAS episodes.

The authors concluded that stressful life events are associated with the onset of RAS but not with the duration, and mental stress had a larger effect than physical stress.

Studies in the past agreed with the results while others have failed to agree with the association. ${ }^{2}$

The causes of RAS are associated with different aetiologies such as hormonal changes, food hypersensitivity, drugs, vitamin deficiencies, bacterial agents, viral agents, local trauma, immune status and environmental factors such as stress. ${ }^{3}$

The problem is how we can use the results from the study as healthcare providers.

Some studies recommend stress-management interventions like relaxation that may be beneficial in reducing RAS recurrence, ${ }^{4}$ and those patients may need supportive psychological management to increase their ability to cope with stress together with the conventional treatment methods. ${ }^{5}$ However some stress can be difficult to predict and to control.

The advice to our patients is that if they have a stressful life event there is a likelihood of the onset of RAS and they should be prepared to handle the episode of RAS. If we could have a magic cure for stress or predict its effects, all we would need is that.

\section{Analia Veitz Keenan and Silvia Spivakovksy} NYU College of Dentistry, New York, USA

1. Lalla RV, Choquette LE, Feinn RS, et al. Multivitamin therapy for recurrent aphthous stomatitis. A randomized, double-masked, placebo-controlled trial. J Am Dent Assoc 2012; 143: 370-376.

2. Picek P, Buljan D, Rogulj AA, et al. Psychological status and recurrent aphthous ulceration. Coll Antropol 2012; 36: 157-159.

3. Natah SS, Konttinen YT, Enattah NS, Ashammakhi N, Sharkey KA, Hayrinen-Immonen R. Recurrent aphthous ulcers today: a review of the growing knowledge. Int J Oral Maxillofac Surg 2004; 33: 221-234.

4. Psychological stress and recurrent aphthous stomatitis. Clinics (Sao Paulo) 2009; 64: 645-648.

5. Albanidou-Farnaki E, Poulopoulos AK, Epivatianos A, Farmakis K, Karamouzis $M$, Antoniades D. Increased anxiety level and high salivary and serum cortisol concentrations in patients with recurrent aphthous stomatitis. Tohoku J Exp Med 2008; 214: 291-296.

Evidence-Based Dentistry (2013) 14, 25. doi:10.1038/sj.ebd.6400919 